Reprod. Nutr. Dévelop., 1986, 26 (2 B), 543-549.

\title{
Absence d'augmentation des récepteurs de la prolactine le jour de l'œstrus dans la glande mammaire de rat nu IPL
}

\author{
Patricia GUILLAUMOT, I. SABBAgh, J. BERTRAND, Hélène COHEN \\ INSERM-U 34, Hôpital Debrousse, 29 rue Sceur Bouvier, 69322 Lyon cedex 05, France. \\ Institut Pasteur, Le Poirier, Lentilly, 69210 L'Arbresle, France.
}

Summary. The prolactin receptors remain constant on the day of estrus in the mammary gland of the IPL nude rat.

Ovine prolactin (o-PRL) binding to rat mammary gland membranes has been shown to vary during the estrous cycle in the normal rat. In this study we report the characteristics of o-PRL binding to mammary gland membranes throughout the estrous cycle in a new rat strain, IPL nude, which presents a total absence of lactation.

Prolactin receptors were quantified in the $100000 \mathrm{~g}$ pellet. The mean value of the affinity constant in IPL nude rat was $16.7 \times 10^{9} \mathrm{M}^{-1}$ and no variation was observed throughout the estrous cycle. The binding capacity also remained unchanged and the values were situated between $11.6 \pm 1.1$. fmoles $/ \mathrm{mg}$ protein and $38.6 \pm 12.3$ fmoles $/ \mathrm{mg}$ protein, corresponding to the lowest values obtained in normal rat. On the day of estrus, the prolactin binding capacity in the mammary gland of the IPL nude rat was significantly different from that of normal rat.

These findings confirm that prolactin certainly plays an important role in the induction or stimulation of the rat mammary gland function.

\section{Introduction.}

L'importance de la prolactine dans le développement de la glande mammaire de rat sur son fonctionnement est bien établie (Forsyth et Hayden, 1977 ; Shiu et Friesen, 1980). Cependant, le mécanisme d'action de la prolactine dans la glande mammaire est encore mal connu à l'exception de la première étape : la fixation de I'hormone sur son récepteur membranaire. Nous avons montré (Guillaumot et al., 1984) que l'affinité et la capacité des récepteurs de la prolactine variaient au cours du cycle œstral dans la glande mammaire de rat. On observe, en particulier, une augmentation importante du nombre des récepteurs de la prolactine le jour de l'estrus ; cette augmentation pourrait être une étape importante dans la préparation des glandes mammaires à la gestation et à la lactation.

Dans cette étude nous décrivons les caractéristiques de la liaison de la prolactine dans les glandes mammaires pendant le cycle œstral d'une nouvelle souche 
de rat, le rat nu IPL, qui présente une absence totale de lactation et nous les comparons à celles des rates normales.

\section{Matériels et méthodes.}

Animaux. - Les rats nus IPL sont élevés à l'Institut Pasteur de Lyon (France) et sont comparés à des rats Sprague-Dawley (rats normaux provenant de l'Institut Mérieux, les Oncins, I'Arbresle, France).

Tous les rats sont gardés dans une animalerie dont les conditions d'éclairage $(12 \mathrm{~h}$ de lumière de $6 \mathrm{~h} 30$ à $18 \mathrm{~h} 30)$ et de température $\left(22^{\circ} \pm 1^{\circ} \mathrm{C}\right)$ sont constantes. Ils recoivent de la nourriture et de l'eau ad libitum.

Des frottis vaginaux quotidiens sont faits pour déterminer le stade du cycle œstral et seuls les animaux présentant au moins deux cycles réguliers de 4 jours sont utilisés.

Le stade du cycle œstral est aussi contrôlé par la détermination des taux sériques de $P R L$, de $L H$ et d'œstradiol $\left(E_{2}\right)$.

Les rats sont décapités entre 10 et $12 \mathrm{~h}$ les jours du diestrus I, diestrus II et de l'estrus, et à $10 \mathrm{~h}, 12 \mathrm{~h}, 16 \mathrm{~h}$ pendant le jour du prœestrus. Pour chaque mesure de la liaison de la prolactine nous avons utilisé les glandes mammaires de 5 rates. A chaque stade du cycle, 3 à 6 mesures ont été faites. Les glandes mammaires inguinales sont immédiatement prélevées, pesées, congelées dans l'azote liquide et stockées à $-70^{\circ} \mathrm{C}$ jusqu'au dosage. Les échantillons de sang sont pris après décapitation et le sérum est stocké à $-20^{\circ} \mathrm{C}$ jusqu'aux dosages.

Fractionnement tissulaire. - Les membranes des glandes mammaires sont préparées selon la méthode de Posner et al. (1974). Le culot membranaire est traité par $\mathrm{MgCl}_{2}, 4 \mathrm{M}$ afin d'enlever la prolactine endogène liée à son récepteur (Kelly et al., 1979).

Mesure de la fixation de la prolactine. - L'ovine prolactine (o-PRL) utilisée est iodée par une méthode à la lactoperoxydase (Thorell et Johansson, 1971) et purifiée comme il a été décrit précédemment (Guillaumot et al., 1984). Son activité spécifique est d'environ $150 \mu \mathrm{Ci} / \mu \mathrm{g}$. Les mesures de la liaison de ${ }^{125} \mathrm{I}-\mathrm{o}-\mathrm{PRL}$ aux membranes de glandes mammaires sont réalisées selon la méthode déjà décrite (Guillaumot et al., 1984).

Les récepteurs de la prolactine sont quantifiés à partir de la représentation de Scatchard (1949). Le nombre des récepteurs ( $N$ max) et la constante d'affinité $\left(K_{A}\right)$ dans la représentation de Scatchard sont obtenus par analyse de la régression linéaire (méthode des moindres carrés). Pour chaque Scatchard, les membranes des glandes mammaires de 5 animaux ont été groupées. De plus, les essais de liaison des rats normaux et des rats nus IPL ont été réalisés simultanément au même stade du cycle œstral.

Dosage de la prolactine (r-PRL) et de l'hormone lutéinisante (r-LH). - La r-PRL et la $\mathrm{r}$-LH ont été mesurées par dosage radioimmunologique selon les instructions données avec les kits du NIAMDD, mais le second anticorps est remplacé par un anticorps en phase solide (DASP provenant de chez ORGANON). Les taux séri- 
ques de $r-P R L$ et de $r-L H$ sont respectivement exprimés en $n g / m l ~ r-P R L-R P_{2}$ et

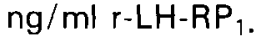

Analyse statistique. - Le test de Student et l'analyse des variances (test ANOVA 1) ont été utilisés pour l'analyse statistique des données ; la différence est considérée comme statistiquement significative quand $p<0,05$.

\section{Résultats.}

Poids des glandes mammaires. - Le poids de la glande mammaire du rat nu IPL ne varie pas pendant le cycle oestral tandis que celui du rat normal augmente et atteint un maximum le jour de l'estrus (tabl. 1).

\section{TABLEAU 1}

Poids des glandes mammaires $(\mathrm{g})$.

\begin{tabular}{|c|c|c|c|c|c|c|}
\hline & PE $10 \mathrm{~h}$ & PE $12 \mathrm{~h}$ & PE $16 \mathrm{~h}$ & Estrus & $\mathrm{DE} I$ & DE $\|$ \\
\hline $\begin{array}{l}\text { Rat normal } . \\
\text { Rat nu IPL . }\end{array}$ & $\begin{array}{l}3,07 \pm 0,21 \\
3,24 \pm 0,15\end{array}$ & $\begin{array}{l}3,18 \pm 0,35 \\
2,57 \pm 0,16\end{array}$ & $\begin{array}{l}2,96 \pm 0,13 \\
2,90 \pm 0,14\end{array}$ & $\begin{array}{l}3,48 \pm 0,23 \\
2,66 \pm 0,08\end{array}$ & $\begin{array}{l}3,19 \pm 0,17 \\
2,78 \pm 0,13\end{array}$ & $\begin{array}{l}3,02 \pm 0,11 \\
3,10 \pm 0,15\end{array}$ \\
\hline $\begin{array}{l}P \\
\text { (rat normal } \\
\text { vs rat nu IPL) }\end{array}$ & NS & NS & NS & $p=0,006$ & NS & NS \\
\hline
\end{tabular}

Chaque valeur est la moyenne $\pm D S M$ de 10 à 15 glandes mammaires.

PE : proestrus ; DE : diestrus.

Taux sériques de $P R L$ et de $\angle H$. - Nous obtenons les valeurs sériques maximales de $r$-PRL et de $r$-LH le jour du proestrus à $16 \mathrm{~h}$ aussi bien chez le rat nu IPL que chez le rat normal (tabl. 2 et 3 ).

Les taux sériques de LH pendant le cycle œestral sont les mêmes chez les deux souches de rat.

Les valeurs sériques de PRL sont significativement plus basses chez le rat nu IPL les jours du diestrus, I, diestrus II et proestrus à $10 \mathrm{~h}$ et $12 \mathrm{~h}$; cependant, la prolactine sérique augmente le jour du prœstrus chez le rat nu IPL comme chez le

TABLEAU 2

Valeurs de PRL dans le sérum des rates au cours du cycle oestral.

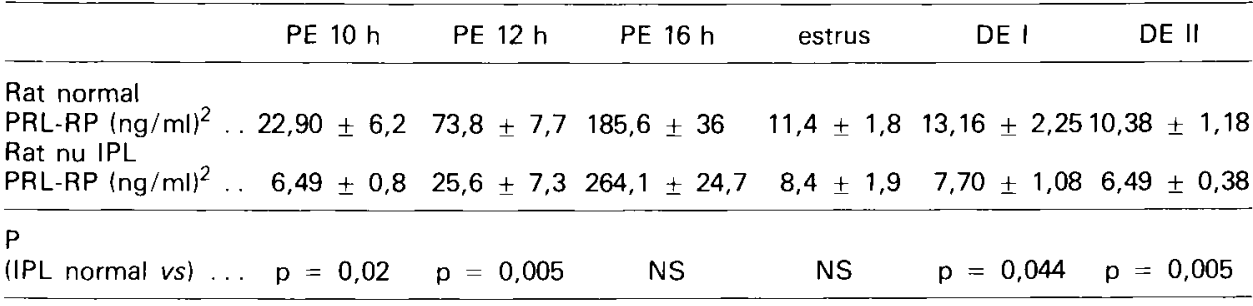

Chaque valeur est la moyenne de 15 à 20 rates \pm D S M 
rat normal et les valeurs ne sont plus différentes à $16 \mathrm{~h}$ et le jour de l'estrus (tabl. 2).

TABLEAU 3

Valeurs de $L H$ dans le sérum des rates au cours du cycle œestral.

\begin{tabular}{|c|c|c|c|c|c|c|}
\hline & PE $10 \mathrm{~h}$ & PE $12 \mathrm{~h}$ & PE $16 \mathrm{~h}$ & Estrus & DE I & DE II \\
\hline $\begin{array}{l}\text { Rat normal } \\
\text { LH-RP }(\mathrm{ng} / \mathrm{ml})^{1} \\
\text { Rat nu IPL } \\
\text { LH-RP }(\mathrm{ng} / \mathrm{ml})^{1}\end{array}$ & $\begin{array}{r}101,2 \pm 3,2 \\
98,9 \pm 5,4\end{array}$ & $\begin{array}{r}104,4 \pm 4,7 \\
95,7 \pm 10,5\end{array}$ & $\begin{array}{l}961,0 \pm 173 \\
662,2 \pm 117,9\end{array}$ & $\begin{array}{l}97,2 \pm 4,9 \\
82,3 \pm 14,4\end{array}$ & $\begin{array}{l}90,3 \pm 5,6 \\
74,3 \pm 10,2\end{array}$ & $\begin{array}{l}93,6 \pm 18,6 \\
99,8 \pm 15,9\end{array}$ \\
\hline (IPL norm & NS & NS & NS & NS & NS & NS \\
\hline
\end{tabular}

Chaque valeur est la moyenne de 15 à 20 rates $\pm D S M$.

Caractéristiques de la liaison de 125/-o-PRL pendant le cycle oestral. - Chez le rat nu IPL, I'analyse de la liaison de la prolactine aux membranes des glandes mammaires pendant le cycle œstral montre:

- l'existence de sites de liaison spécifiques et saturables ;

- la constante d'affinité $\left(K_{A}\right)$ de la liaison de ${ }^{125}$-o-PRL aux membranes des glandes mammaires ne varie pas pendant le cycle œstral (tabl. 4). Chez le rat nu IPL, la constante d'affinité moyenne au cours du cycle est de $16,7 \times 10^{9} \mathrm{M}^{-1}$.

- Le nombre des récepteurs ( $\mathrm{Nmax}$ ) de la prolactine dans les glandes mammaires de rat nu IPL ne varie pas non plus pendant le cycle œestral ; la capacité, comprise entre $11,6 \pm 1,1 \mathrm{fmoles} / \mathrm{mg}$ de protéines et $38,6 \pm 12,3 \mathrm{fmoles} / \mathrm{mg}$ de protéines correspond aux valeurs les plus basses obtenues chez le rat normal (fig. 1). Le

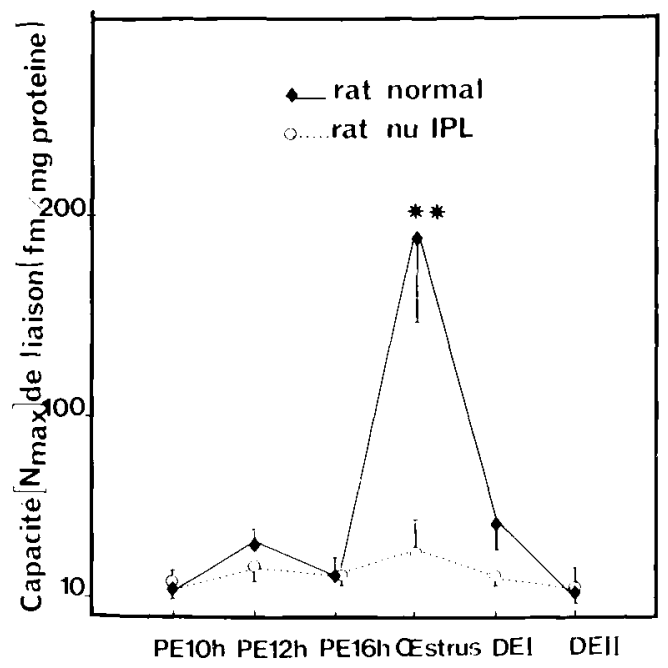

FIG. 1. - Variation du nombre des récepteurs de la prolactine (exprimé en fmoles de prolactine liée par $\mathrm{mg}$ de protéine membranaire) pendant le cycle cestral chez le rat nu IPL 10 ) et chez le rat normal $(\bullet)$. Chaque valeur est la moyenne $+D S M$ de 4 à 6 mesures. 
jour de l'estrus, le nombre des récepteurs de la prolactine dans les glandes mammaires du rat nu IPL n'augmente pas comme nous l'avons montré chez le rat normal. A ce moment le Nmax chez le rat nu IPL diffère de façon très significative de celui du rat normal (fig. 1).

\section{TABLEAU 4}

Liaison de ${ }^{125}$-oPRL aux membranes de glandes mammaires de rat pendant le cycle cestral: valeurs des constantes d'affinité $\left(10^{9} \mathrm{M}^{-1}\right)$.

\begin{tabular}{lccrr}
\hline & Rat normal & $\begin{array}{c}\mathrm{P} \\
\text { (estrus vs } \\
\text { autres stades) }\end{array}$ & Rat nu IPL & \multicolumn{1}{c}{$\begin{array}{c}\mathrm{P} \\
\text { (estrus vs } \\
\text { autres stades) }\end{array}$} \\
\cline { 2 - 5 } PE 10 h & $15,0 \pm 0,5$ & $\mathrm{p}=0,002$ & $22,3 \pm 19,4$ & $\mathrm{NS}$ \\
PE 12 h & $19,6 \pm 6,7$ & $\mathrm{p}=0,019$ & $18,5 \pm 5,5$ & $\mathrm{NS}$ \\
PE 16 h & $20,3 \pm 2,2$ & $\mathrm{p}=0,0001$ & $16,6 \pm 6,4$ & $\mathrm{NS}$ \\
Estrus & $5,03 \pm 1,34$ & $\mathrm{NS}$ & $16,0 \pm 5,2$ & $\mathrm{NS}$ \\
DE I & $5,15 \pm 1,22$ & $\mathrm{~N}$ & $8,5 \pm 4,2$ & $\mathrm{NS}$ \\
DE II & $18,2 \pm 2,13$ & $\mathrm{p}=0,020$ & $18,6 \pm 12,0$ & $\mathrm{NS}$ \\
\hline
\end{tabular}

Chaque valeur donnée est la moyenne \pm D S M de 4 à 6 déterminations différentes.

Chaque détermination a été faite à partir d'un groupe de 5 rats.

\section{Discussion.}

Cette étude a montré que, chez le rat nu IPL, caractérisé par une absence totale de lactation, les récepteurs de la prolactine ne varient pas pendant le cycle œstral tandis que, chez le rat normal, nous avons observé des variations dans le nombre des sites de liaison et dans leur affinité. Chez le rat nu IPL, les paramètres sont significativement différents de ceux du rat normal le jour de l'estrus, et les valeurs obtenues sont similaires à celles du rat normal le jour du diestrus II ou du proestrus. Le poids des glandes mammaires est aussi significativement différent entre les deux souches le jour de l'estrus.

La prolactine est connue pour augmenter ses propres récepteurs dans le foie (Costlow et al., 1976; Posner, 1976; Manni et al., 1978) et dans les glandes mammaires (Bohnet et al., 1977 ; Djiane et Durand, 1977; Hayden et al., 1979) dans de nombreuses expérimentations ou situations physiologiques. Bien que le taux sérique de la prolactine augmente pendant le prostrus chez le rat nu IPL nous n'observons pas d'augmentation du nombre des récepteurs de la prolactine le jour de l'estrus. Ce résultat pourrait peut-être s'expliquer par la diminution de la bioactivité de la prolactine sérique du rat nu IPL qui a été observée (Cohen et al., 1983). D'autre part, l'effet stimulant de la prolactine sur ses propres récepteurs dans la glande mammaire passerait par une augmentation du nombre des cellules épithéliales (Hayden et al., 1979). Chez le rat normal, le poids des glandes mammaires augmente effectivement le jour de l'estrus tandis que chez le rat nu IPL aucune variation n'est observée pendant le cycle œstral et le jour de l'estrus la différence entre les deux souches est significative. 
En outre, l'action de la prolactine dans le contrôle de ses propres récepteurs pourrait être médiée par un ou plusieurs autres facteurs :

- L'cestradiol est connu pour augmenter les récepteurs de la prolactine dans les glandes mammaires (Hayden et al., 1979), cependant il a été démontré que l'effet œstrogénique sur les récepteurs de prolactine était probablement dû, à la prolactine elle-même, puisque cet effet est bloqué par hypophysectomie et partiellement restauré par des injections de prolactine. Quoi qu'il en soit, les taux sériques d'œstradiol, chez le rat nu IPL pendant le cycle œstral, sont semblables à ceux du rat normal (Sabbagh et al., 1984).

- La progestérone est connue (Djiane et Durand 1977 ; Hayden et al., 1979) pour antagoniser les effets stimulateurs de la prolactine et des œstrogènes sur les récepteurs lactogéniques dans les glandes mammaires. Elle a aussi été écrite pour être impliquée dans la croissance et la différenciation de la glande mammaire (Dulbecco et al., 1982). Cependant, le taux sérique de progestérone chez le rat nu IPL n'est pas significativement différent de celui du rat norrnal pendant le cycle œestral (Sabbagh et al., 1984).

Parmi les facteurs connus agissant sur l'induction des récepteurs de la prolactine, seule la prolactine a été trouvée déficiente aussi bien quantitativement que qualitativement chez le rat nu IPL. Cependant, la prolactine pourrait aussi agir indirectement en augmentant un autre facteur. Son action pourrait aussi être potentialisée par un facteur indépendant ; un tel facteur appelé "Synlactin 》 a été récemment décrit par Nicoll et al. (Anderson et al., 1983 ; Nicoll et al., 1984, $1985)$ : il potentialise l'action de la prolactine sur la multiplication cellulaire de la glande mammaire de rat.

Quel que soit le mécanisme qui régule les récepteurs de la prolactine, ces résultats confirment que la prolactine joue certainement un rôle important dans la croissance et le fonctionnement de la glande mammaire.

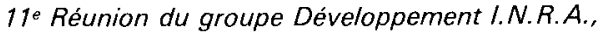
Montpellier, 22-24 mai 1985.

Remerciements. - Nous remercions le NIAMDD qui nous a fourni gracieusement les kits pour les dosages radioimmunologiques et la prolactine ovine. Nous remercions aussi $\mathrm{M}$. Montagnon pour son excellent travail de secrétariat.

\section{Références}

ANDERSON T. R., RODRIGUEZ J., SPENCER E. M., NICOLL C. S., 1983. The synlactin hypothe sis : a somatomedin-like molecule may be involved with prolactin's mitogenic action. 65 th annu. Meet. Endocr. Soc., Abstr. 350.

BOHNET H. G., GOMEZ F., FRIESEN H. G., 1977. Prolactin and estrogen binding sites in the mammary gland of the lactating and non-lactating rat. Endocrinology, 101, 1111-1121.

COHEN H., SABBAGH I., GUILLAUMOT P., ROBERTSON M., FRIESEN H. G., BERTRAND J., 1983. Relationships between bioassay and RIA measurement of prolactin in genetically hypoprolactinemic rat : IPL nude. 65th annu. Meet. Endocr. Soc., Abstr. 625.

COSTLOW M. E., BUSCHOW R. A., McGUIRE W. L., 1976. Prolactin stimulation of prolactin receptors in rat liver. Life Sci., 17, 1457-1466.

DJIANE J., DURAND P., 1977. Prolactin-progesterone antagonism in self regulation of prolactin receptors in the mammary gland. Nature, 266, 641-643. 
DULBECCO R., HENAHAN M., ARMSTRONG B., 1982. Cell types and morphogenesis in the mammary gland. Proc. nat. Acad. Sci. USA, 79, 7346-7350.

FORSYTH I. A., HAYDEN T. J., 1977. Comparative endocrinology of mammary growth and lactation. Symp. Zool. Soc. Lond., 41, 135-163.

GUILLAUMOT P., SABBAGH I., BERTRAND J., COHEN H., 1984. Prolactin receptors in the rat mammary gland. Change during the estrous cycle. Biochem. biophys. Res. Comm., 123, 612617.

HAYDEN T. J., BONNEY R. C., FORSYTH I. A., 1979. Ontogeny and control of prolactin receptors in the mammary gland and liver of virgin, pregnant and lactating rats. J. Endocrinol., 80, $259-269$.

KELLY P. A., LEBLANC G., DJIANE J., 1979. Estimation of total prolactin - binding sites after in vitro desaturation. Endocrinology, 104, 1631-1638.

MANNI A., CHAMBERS M. J., PEARSON O. H., 1978. Prolactin induces its own receptors in rat liver. Endocrinology, 103, 2168-2171.

NICOLL C. S., ANDERSON T. R., HEBERT N., SPENCER E. M., 1984. Comparative aspects of prolactin's growth promoting actions : evidence for synergism with an insulin-like growth factor (IGF)/somatomedin (SM). Nth int. Congr. Prolactin, Abstr. 85.

NICOLL C. S., HEBERT N. J., RUSSEL S. M., 1985. Lactogenic hormones stimulate the liver to secrete a factor that acts synergistically with prolactin to promote growth of the pigeon cropsac mucosal epithelium in vivo. Endocrinology, 116, 1449-1453.

POSNER B. I., 1976. Regulation of lactogen specific binding sites in rat liver : studies on the role of lactogens and estrogens. Endocrinology, 99, 1168-1177.

POSNER B. I., KELLY P. A., SHIU R. P. C., FRIESEN H. G., 1974. Studies of insulin, growth hormone and prolactin binding : tissue distribution, species variation and characterization. Endocrinology, 95, 521-531.

SABBAGH I., GUILLAUMOT P., BERTRAND J ., COHEN H., 1984. The hypoprolactinemic rat (IPL) : pituitary and sex steroid hormones during the estrous cycle and gestation. 7 th int. Congr. Endocrinol., Abstr. 2133.

SCATCHARD G., 1949. The attraction of protein for small molecules and ions. Ann. N. Y. Acad. Sci., 51, 660-672.

SHIU R. P. C., FRIESEN H. G., 1980. Mechanism of action of prolactin in the control of mammary gland function. Ann. Rev. Physiol., 42, 83-96.

THORELL J. I., JOHANSSON B. G., 1971. Enzymatic iodination of polypeptides with ${ }^{125}$ I to high specific activity. Biochim. biophys. Acta, 251, 363-369. 